Accelerator Division

Alternating Gradient Synchrotron Department

BROOKHAVEN NATIONAL LABORATORY

Upton, New York 11973

Accelerator Division

Technical Note

AGS/AD/Tech. Note No. 384

EVOLUTION OF A WORKSTATION-BASED CONTROL

SYSTEM AT THE BNL AGS -- THE

PATH TO STANDARDS COMPLIANCE*

J.F. Skelly

November 5, 1993

*Presentation made at the Workshop on Workstations \& Software Tools for Automatic Control '93, Prague, Czech Republic, 10/26-27/93. 
Workshop on Workstations \& Software Tools for Automatic Control '93 Prague, Czech Republic 10/26-27/93.

\title{
EVOLUTION OF A WORKSTATION-BASED CONTROI SYSTEM AT THE BNL AGS - THE PATH TO STANDARDS COMPLIANCE
}

\author{
J.F. Skelly \\ AGS Department \\ Brookhaven National Laboratory \\ Upton, New York 11973 USA
}

\begin{abstract}
A workstation-based computerized control system was installed at the Brookhaven AGS beginning in 1985 with a few nodes for developmental purposes, and expanded into the AGS Distributed Control System (AGSDCS) during the Booster project (1988-92). The AGSDCS now comprises about 100 workstation nodes on both Ethernet and Domain Token Ring networks. The AGSDCS began as a homogeneous network of Apollo workstations, using Apollo-standard software tools and networking techniques, with an underlying proprietary data network for communication with accelerator equipment interfaces. The AGS experience suggests the necessity for a heterogeneous network and compliance with widely-supported software and hardware standards; a majority of the original AGs controls design decisions now have been reconsidered. This paper discusses the on-going effort to integrate standards-compliant software tools into the AGS controls environment, illustrating both the successes and mistakes of the original AGSDCS design.
\end{abstract}

\section{1 - Introduction}

It was only two years ago (at ICALEPCS'91) that the accelerator controls community realized that it had a "standard model" for an accelerator control system, a model which prescribes a network with workstations serving as operator consoles and real-time systems serving as Front End Computers (FECs). The AGS at Brookhaven has had a control system roughly conforming to the standard model for about eight years. The AGS Controls staff currently commits a significant fraction of its programming resources to maintenance efforts, which largely have the nature of upgrade projects. A study has been undertaken of completed, ongoing, and contemplated upgrade projects to examine the forces driving these high maintenance requirements. Fundamentally, all these upgrade projects are efforts to evolve the control system away from dependence on narrowly supported or proprietary standards, and towards broadly supported standards.

A summary of these upgrade projects follows, and some discussion of the larger trends driving them. A list of the "final" standards towards which the AGS (and RHIC) control systems currently aim is presented. Finally, it is suggested that the goal of software sharing among accelerators, which has gained considerable support recently, should be pursued by first developing some consensus on the (broadly supported) controls standards to which the shared software should conform. note.

The slides presented at the WSTAC'93 workshop are appended to this 


\section{2 - Standards Evolution}

Typically, there are two basic reasons why it becomes desirable to modify a control system in favor of a new standard. Either the new standard offers some improvement to the control system, such as enhanced reliability or performance, or the modification is mandated by a critical vendor in the course of an upgrade to its computer line, network support, or operating system. A controls group may consider two strategies to minimize the number of such modifications it is obliged to support.

The first strategy is to rely on a very strong (and large) vendor, and hope that the vendor will undertake all the work involved in supporting new standards, while preserving unchanged the interface to the controls group software. However, even this strategy has its limits; even a vendor as large as DEC cannot promise indefinitely to shield a customer from such market forces as Unix or Windows NT.

A second strategy is for the controls group to carefully evaluate the standards it chooses to adopt, and select only standards which are broadly supported (multiple vendors) and which offer hope, by their market penetration, both of long term survival and of substantial lead time if they finally must be abandoned.

The experience at the AGS has indicated that standards evolution has occurred in four basic areas:

Network

Graphic User Interface (GUI)

Operating System (OS)

File System

\section{3 - AGS Evolution}

The AGS experience with the standard model began with a variant of the usual network design. The FECs were segregated onto a proprietary network (Relway) which was attached to the (homogeneous) Apollo workstation network via gateways (Slide 2). The FECs themselves comprised two components, the "station" and the "controller", each constructed from 8086 generation Intel single board computers; the station and controller levels communicated over GPIB using a simple locally developed protocol.

The evolution from this beginning is summarized in slide 3 . completed projects are indicated by checks, on-going projects by squares, and future projects by circles.

For the Booster Project, the Intel station was replaced by one constructed from an Apollo workstation, and these stations were attached directly to the Domain Token Ring network; the communication protocol between the station and application software was then implemented atop Apollo Mailboxes. Subsequently, the network was expanded to include Ethernet segments, and Apollo stations were introduced on the Ethernet (Dibbuk project). The most recently completed project involved abandonment of the Apollo Mailbox for station-application communication, and introduction of a protocol based on SUN Remote Procedure Calls (RPCs). 
Ongoing upgrade projects involve substantial work to evolve away from Apollo's proprietary GUI and towards the widely supported $\mathrm{X}$ windows and Motif system. We are also developing a VME-based FEC as an alternative to the Apollo station (which is not a satisfactory real-time platform); this new FEC offers the option of integrating the station and controller layers into a single tightly coupled system. This FEC design is very close to that under development for the RHIC project, and in particular shares extensively hardware and software components with RHIC work.

Future projects involve adoption of the Network File System (NFS) and of a true Unix operating system. Much of this upgrade work is motivated by the fact that Hewlett Packard has terminated development of the Apollo workstation line, and future acquisitions of more powerful workstations will target UNIX RISC workstations. The AGS at that point must support a heterogeneous network, with both Apollo and UNIX nodes interoperating. A careful choice of standards for this heterogeneous network can make it possible to support workstations from multiple vendors in this environment as well.

Additional upgrade projects will be necessary to support a heterogeneous network; the current use of "station" protocol layered on top of RPC does not take advantage of the RPC capability to perform type conversion in a heterogeneous network, and should be replaced by a more native use of the RPC protocol.

In the arena of services, the issue of database support must be addressed as the Apollo platforms become obsolete; the anticipated use of Sybase in the RHIC project suggests that even the choice of Interbase should not be regarded as irrevocable.

\section{4 - AGS (\& RHIC) STANDARDS}

The final slide presents the list of standards which the AGS (and RHIC) control group proposes as a viable base for development of robust control systems. In a workshop such as this, this list is expected to meet with discussion and even dissent, but it is suggested that such discussion is prerequisite for any serious effort to share software solutions among different laboratories.

The list speaks for itself, but it is worth mention that the RPC protocol is specifically selected not only for its broad support in industry, but also because it offers considerable promise for development of communication protocols that support the current trend for construction of FECs that implement object oriented architectures. Such FECs are in use at the AGS as well as at a number of other laboratories; an important issue then is the provision of a protocol for efficient communication between the FEC and application software, one which exports a view of the object oriented architecture in the FEC. 


\section{AGS DISTRIBUTED CONTROL SYSTEM}

8 Years Experience (1985 - 1993)

Current high maintenance burden

$\square$ narrowly supported (or proprietary)

$\rightarrow$ broadly supported standards

$\bigcirc$ Our Convenience

(reliability, efficiency)

$\bigcirc$ Vendor Enforced

$\square$ Strategies:

Choice of Vendor

Choice of Standards

$\square$ Maintenance Projects

Network

GUI

O/S

File Sys

$\square$ Standards

$\square$ Impact on Software Sharing 


\section{DOMAIN TOKEN RING}

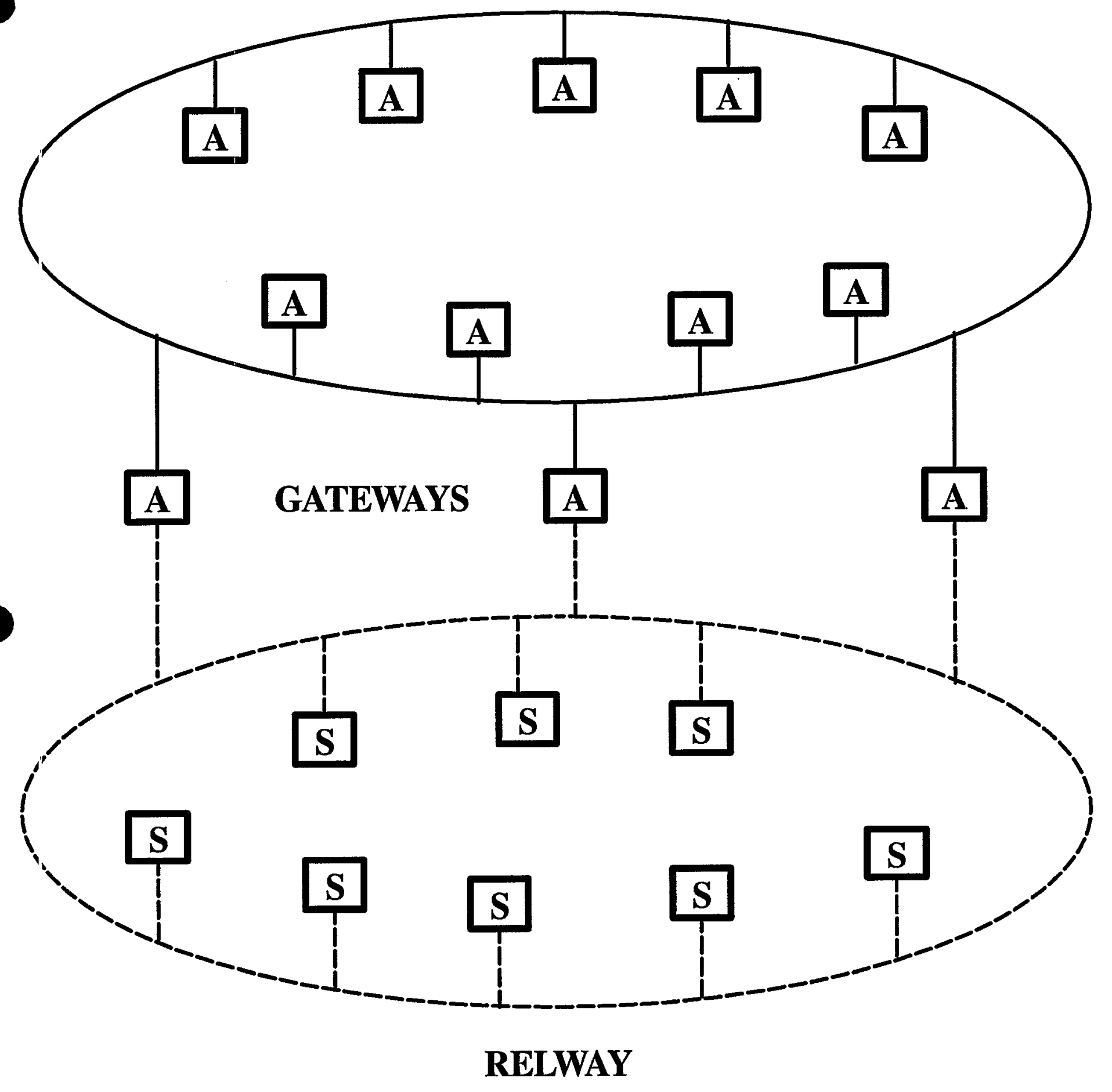

A Apollo

S Station $($ Intel SBC) $=$ FEC 
$\checkmark$ FEC Network:

$\checkmark$ FEC:

FEC Protocol:

FEC Network:

FEC Protocol:

FEC:

GUI Wind Man: Display Man.

Graphics Toolkit: GPR

GUI Toolkit:
RELWAY

Intel SBC

"Station"

Token Ring

Station/Mbx

Apollo

Dialogue/GPR
- Domain Token Ring

- Apollo

- Station/Apollo-Mbx

Ethernet

- Station/RPC

- VMEbus

1 Motif/X W.M.

- Xlib

Motif Widgets

GUI Libraries: Dialogue/GPR $\quad$ Motif Widgets (MenuBar, MenuList, QG, Generic Popups)

O File System:

Apollo

$\rightarrow$ NFS

Op System:

Aegis

UNIX (OSF?)

Host Network: homogeneous

$\rightarrow$ heterogeneous

(Apollo 68K) (HP 68K, HP RISC) $(+? ?)$

O FEC Protocol: Station/RPC $\quad$ Native RPC

Services:
Video:
RGB/ATbus
- VGA/VME
○ Database:
Interbase/Apollo
? 


\section{AGS (\& RHIC) STANDARDS}

Network:

Ethernet

TCP/IP

RPC

FEC: Object-Oriented Architecture

O/S:
Unix (OSF?) / Workstation
vxWorks / VMEbus

GUI:

$\mathbf{X}$

Motif Window Manager

Motif Widgets

File System:

NFS 